\title{
On a steady flow of multicomponent, compressible, chemically reacting gas
}

\author{
Ewelina Zatorska
}

May 22, 2012

\author{
Institute of Applied Mathematics and Mechanics \\ University of Warsaw \\ ul. Banacha 2, 02-097 Warszawa, Poland \\ E-MAIL: E.ZATORSKA@MIMUW.EDU.PL
}

\begin{abstract}
We consider the system of equations governing a steady flow of polyatomic isothermal reactive gas mixture. The model covers situation when the pressure depends on species concentration and when the diffusion coefficients for each of the species are density-dependent. It is shown that this problem admits weak solution provided the adiabatic exponent for the mixture $\gamma$ is grater then $\frac{7}{3}$.

Keywords: multicomponent flow, chemically reacting gas, steady compressible NavierStokes system, weak solution.

Mathematics Subject Classification (2000). 35B45, 35D40, 76N10, 35Q30
\end{abstract}

\section{Introduction}

There is a strong motivation to investigate the equations describing motion of multicomponent reactive flows. Such models arise in many fields of science: astrophysics, chemistry, mathematical biology and in the engineering applications: modelling combustion, chemical reactors, pollutant formation in the atmosphere or crystal growth. These phenomena require to deal with equations of very complex mathematical structure and properties. Because of a huge gap in nowadays available methods one must look for simplified models that can still be applied in various situations. In this work we capture the case of steady motion for the mixture of 4 compressible gas-components undergoing an isothermal, reversible chemical reaction constituted by

$$
A+B \rightleftharpoons C
$$

and we assume that the reaction takes place in the presence of a dilutant denoted by $D$. Our postulate that the process occurs in a constant temperature is met when the reaction is slow enough to enable the surroundings to continually compensate the difference in heats between the reactants and products. Accordingly, our model can be 
characterised by the state variables: the total mass density $\varrho=\varrho(x)$, the velocity vector field $\mathbf{u}=\mathbf{u}(x)$ and the species mass fractions $Y_{k}$ for $k \in S=\{A, B, C, D\}$. The motion of gas is described by the Navier-Stokes equations, which represent the conservation of mass, momentum and the conservation of species mass (see [10], Chapter 2):

$$
\begin{gathered}
\operatorname{div}(\varrho \mathbf{u})=0, \\
\operatorname{div}(\varrho \mathbf{u} \otimes \mathbf{u})-\operatorname{div} \mathbb{S}+\nabla \pi=\varrho \mathbf{f} \\
\operatorname{div}\left(\varrho Y_{A} \mathbf{u}\right)+\operatorname{div}\left(\mathcal{F}_{A}\right)=\varrho \omega_{A}, \\
\operatorname{div}\left(\varrho Y_{B} \mathbf{u}\right)+\operatorname{div}\left(\mathcal{F}_{B}\right)=\varrho \omega_{B}, \\
\operatorname{div}\left(\varrho Y_{C} \mathbf{u}\right)+\operatorname{div}\left(\mathcal{F}_{C}\right)=\varrho \omega_{C}
\end{gathered}
$$

where $\mathbb{S}$ is the viscous stress tensor, $\pi$ denotes the internal pressure of the fluid, $\mathbf{f}$ - external force, $\omega_{k}$ stands for the production rate of the $k$-th species and by $\mathcal{F}_{k}$, $k \in S$ we denote the diffusion fluxes.

We remark that the model is consistent with the principle of mass conservation, thus necessarily

$$
\sum_{k \in S} Y_{k}=1
$$

and

$$
\sum_{k \in S} \omega_{k}=0
$$

Furthermore observe that we consider only the first 3 mass fractions as unknowns and use (3) to evaluate the mass fraction of the remaining species $Y_{D}$, while the dilutant mass flux is given by $\mathcal{F}_{D}=-\mathcal{F}_{A}-\mathcal{F}_{B}-\mathcal{F}_{C}$.

The form of viscous stress tensor $\mathbb{S}$ is determined by the Newton's rheological law as

$$
\mathbb{S}(\mathbf{u})=\mu\left(\nabla \mathbf{u}+(\nabla \mathbf{u})^{T}\right)+\nu \operatorname{div} \mathbf{u} \mathbb{I}
$$

where $\mu, \nu$ are the Lamé viscosity coefficients satisfying the relation

$$
\mu>0, \quad 2 \mu+3 \nu \geq 0 .
$$

The species mass fluxes $\mathcal{F}_{k}, k \in S$, are given by the Fick's empirical law

$$
\mathcal{F}_{k}=-D_{k}(\varrho) \nabla Y_{k}, \quad k \in\{A, B, C\},
$$

where $D_{k}(\varrho)$ stands for the diffusion coefficient

$$
\underline{D}\left(1+\varrho^{\frac{\gamma}{2}}\right) \leq D_{k}(\varrho) \leq \bar{D}\left(1+\varrho^{\frac{\gamma}{2}}\right),
$$

for some positive constants $\underline{D}, \bar{D}$.

The production rates $\omega_{k}$ are usually approximated by the continuous function proportional to the absolute temperature and concentrations of substrates and products. For the isothermal processes we can assume that for $k \in\{A, B, C\}$ we have

$$
-\underline{\omega} \leq \omega_{k}\left(Y_{A}, Y_{B}, Y_{C}, Y_{D}\right) \leq \bar{\omega}, \quad \text { for all } \quad 0 \leq Y_{k} \leq 1,
$$


moreover, we suppose

$$
\omega_{k}\left(Y_{A}, Y_{B}, Y_{C}\right) \geq 0 \quad \text { whenever } Y_{k}=0 .
$$

The system (2) is supplemented by the impermeability conditions

$$
\left.\mathbf{u} \cdot \mathbf{n}\right|_{\partial \Omega}=\left.\mathcal{F}_{k} \cdot \mathbf{n}\right|_{\partial \Omega}=0
$$

together with the no-slip boundary condition

$$
\mathbf{u} \times\left.\mathbf{n}\right|_{\partial \Omega}=\mathbf{0} .
$$

The internal pressure $\pi$ is a function of density $\varrho$ and mass fractions $Y=\left\{Y_{A}, Y_{B}, Y_{C}, Y_{D}\right\}$ and it obeys the following equation of state

$$
\pi(\varrho, Y)=\varrho^{\gamma}+R \varrho\left(\sum_{k \in S} \frac{Y_{k}}{m_{k}}\right), \quad \gamma>1,
$$

where $R$ is the perfect gas constant. The first term describes the elastic pressure while the latter summand represents the thermodynamic pressure for the mixture of 4 species given by Boyle's law. Note that, due to Thomas-Fermi-Weizsäcker approximation, the quantum part of elastic pressure is proportional to $\varrho^{\frac{5}{3}}$, cf. [8].

Our main result concerns the existence of weak solutions in the sense specified by the definition

Definition 1.1 We say a triple of functions $(\varrho, \mathbf{u}, Y)$ is a weak solution to the problem (2), (8-9) provided $\varrho \in L^{\gamma}(\Omega), \mathbf{u} \in W_{0}^{1,2}(\Omega), Y \in W^{1,2}(\Omega),\left.\mathcal{F}_{k} \cdot \mathbf{n}\right|_{\partial \Omega}=0, Y_{k}, \varrho \geq 0$ and $\sum_{k \in S} Y_{k}=1$ a.e. in $\Omega$, and the following integral equalities hold

$$
\begin{gathered}
\int_{\Omega} \varrho \mathbf{u} \cdot \nabla \xi \mathrm{d} x=0, \quad \forall \xi \in C^{\infty}(\bar{\Omega}) \\
\int_{\Omega}(-\varrho(\mathbf{u} \otimes \mathbf{u}): \nabla \varphi+\mathbb{S}(\mathbf{u}): \nabla \varphi) \mathrm{d} x-\int_{\Omega} \pi(\varrho, Y) \operatorname{div} \varphi \mathrm{d} x=\int_{\Omega} \varrho \mathbf{f} \cdot \varphi \mathrm{d} x \quad \forall \varphi \in C_{0}^{\infty}(\Omega) \\
-\int_{\Omega} \varrho \mathbf{u} Y_{k} \cdot \nabla \phi \mathrm{d} x+\int_{\Omega} D_{k}(\varrho) \nabla Y_{k} \cdot \nabla \phi \mathrm{d} x=\int_{\Omega} r_{k} \omega \phi \mathrm{d} x, \quad \forall \phi \in C^{\infty}(\bar{\Omega}) .
\end{gathered}
$$

for all $k \in\{A, B, C\}$.

We will also use the notion of the renormalized solution to the continuity equation

Definition 1.2 Let $\mathbf{u} \in W_{\text {loc }}^{1,2}\left(\mathbb{R}^{3}\right)$ and $\varrho \in L_{\text {loc }}^{6 / 5}\left(\mathbb{R}^{3}\right)$ solve

$$
\operatorname{div}(\varrho \mathbf{u})=0
$$

in the sense of distributions on $\mathbb{R}^{3}$, then the pair $(\varrho, \mathbf{u})$ is called a renormalized solution to the continuity equation, if

$$
\operatorname{div} b(\varrho) \mathbf{u}+\left(\varrho b^{\prime}(\varrho)-b(\varrho)\right) \operatorname{div} \mathbf{u} \mathrm{d} x=0,
$$

in the sense of distributions on $\mathbb{R}^{3}$, for all $b \in W^{1, \infty}(0, \infty) \cap C^{1}([0, \infty))$, such that $s b^{\prime}(s) \in L^{\infty}(0, \infty)$. 
Theorem 1.3 Let $\Omega \in C^{2}$ be a bounded domain in $\mathbb{R}^{3}$, let $\mathbf{f} \in L^{\infty}(\Omega)$. Let $\mu>0$, $2 \mu+3 \nu>0, \gamma>\frac{7}{3}$. Then there exists a weak solution to the problem (2), (8-9) in the sense of Definition 1. Moreover $\int_{\Omega} \varrho \mathrm{d} x=M$, and if $\gamma \geq 3$, then $\varrho \in L^{2 \gamma}(\Omega)$, otherwise if $\frac{7}{3}<\gamma<3$ then $\varrho \in L^{3 \gamma-3}(\Omega)$. Additionally, the pair $(\varrho, \mathbf{u})$ is a renormalized solution to the continuity equation in the sense of Definition 2.

The proof of this fact relies on some ideas from the theory of weak solutions to the Navier-Stokes equations for compressible fluids, which is mainly due to the pioneering work of Lions [13] and Feireisl [8]. An overview of these results is given in the monograph [19]. A modification of this approach in case of steady flows with slip boundary conditions has been introduced by Mucha and Pokorný in a two dimensional case in [14] and in 3D in [20].

Much less is known about the problems that model additionally chemical reactions. For the evolutionary case the existence of global in time weak solutions to the system supplemented by the equation expressing the conservation of energy, and physically relevant constitutive relations was established by Giovangigli [10]. He assumed, however, that the initial conditions are sufficiently close to an equilibrium state. A first rigorous proof of existence of the, so-called, weak variational entropy solutions is due to Feireisl, Petzeltová and Trivisa [7]. Unfortunately it seems that their approach can not be applied to the case when the pressure depends on the species concentration, mainly because of rather complex balance law for the entropy. Regarding simplified models, the situation presents better, especially for one dimensional models that were studied in a series of articles [6], [21], [2], and for the multidimensional combustion models, for which the global existence of weak solutions with large initial data was recently presented in [4] and then extended in [5] to treat the pressure dependence on the mass fraction of the reactant.

From our point of view investigations devoted to stability and asymptotic analysis of multicomponent reactive flows [11], [12] are of particular interest, since, to the best of our knowledge, the steady problems were not considered so far. They seem to be worse than the non steady cases in the sense that the energy inequality by itself does not give any information about the sequence of weak solutions. Nevertheless, the recent works of Mucha, Pokorný [15], [16] and Novotný, Pokorný [17], [18] shed some light on the issue of existence of solutions to the steady Navier-Stokes-Fourier system, which makes obtainment of similar results for multicomponent reactive flows more likely. Therefore the main objective of the present paper is to undertake a first step in this direction.

Let us now outline the strategy of the proof, and thus the structure of the paper. In the next section we introduce the approximative system following the approach from [19], [7]. At this stage the classical theory for elliptic equations together with the fixed point argument are sufficient to show the existence of regular solutions. In this section we also show the basic energy estimate that is used, with some modifications, throughout all the paper. Next we let $\varepsilon \rightarrow 0$ in order to get rid of artificial viscosity. The requirement imposed on the heat capacity ratio, $\gamma>\frac{7}{3}$ is necessary to get the boundedness of velocity gradient in $L^{2}(\Omega)$ and enables to apply Lions technique of showing the strong convergence of density. The only changes are caused by the part of 
pressure that depends on the species concentration.

\section{Approximation}

Combining the ideas from [19] and [7] we introduce the following approximative system. For the constant parameters $h, \varepsilon, \eta>0$ we will look for a triple $\left(\varrho_{\eta, \varepsilon}, \mathbf{u}_{\eta, \varepsilon}, Y_{\eta, \varepsilon}\right)$ (we will skip the subscripts when no confusion can arise) satisfying:

- the approximate continuity equation

$$
\begin{gathered}
\varepsilon \varrho+\operatorname{div}(\varrho \mathbf{u})=\varepsilon \Delta \varrho+\varepsilon h \\
\left.\nabla \varrho \cdot \mathbf{n}\right|_{\partial \Omega}=0
\end{gathered}
$$

- the approximate momentum equation

$$
\begin{gathered}
\frac{1}{2} \varrho \mathbf{u} \cdot \nabla \mathbf{u}+\frac{1}{2} \operatorname{div}(\varrho \mathbf{u} \otimes \mathbf{u})-\operatorname{div} \mathbb{S}(\mathbf{u})+\nabla \pi(\varrho, Y)=\varrho \mathbf{f} \\
\left.\mathbf{u}\right|_{\partial \Omega}=0
\end{gathered}
$$

- the approximate species balance equations

$$
\begin{gathered}
\varrho \mathbf{u} \cdot \nabla Y_{k}-\operatorname{div}\left(\left(D_{k}\right)_{\eta} \nabla Y_{k}\right)=\varrho \omega_{k}, \quad k \in\{A, B, C\} \\
\left.\nabla Y_{k} \cdot \mathbf{n}\right|_{\partial \Omega}=0,
\end{gathered}
$$

where $\left(D_{k}\right)_{\eta}$ is a smooth approximation of $D_{k}(\varrho)$. In particular, regularised diffusion coefficients conserve the property (5) uniformly with respect to $\eta$.

The aim of this section is to prove

Theorem 2.1 Let $\epsilon>0, h=\frac{M}{|\Omega|}$. Under assumptions of Theorem 1, there exists a triple $(\varrho, \mathbf{u}, Y)$ being a regular solution to $(13,14,15)$, such that $\varrho \in W^{2, p}(\Omega), \mathbf{u} \in$ $W^{2, p}(\Omega), Y_{k} \in W^{2, p}(\Omega), k \in S$, for all $p<\infty$. Moreover, $\varrho \geq 0$ in $\Omega, \int_{\Omega} \varrho \mathrm{d} x=M$, $Y_{k} \geq 0$ and $\sum_{k \in S} Y_{k}=1$.

In the first step we denote for $p \in[1, \infty]$ :

$$
M^{p}=\left\{\mathbf{w} \in W^{1, p}(\Omega) ;\left.\mathbf{w}\right|_{\partial \Omega}=\mathbf{0}\right\},
$$

and define the operator

$$
\mathcal{S}: M^{\infty} \rightarrow W^{2, p}(\Omega),
$$

$1 \leq p<\infty, \mathcal{S}(\mathbf{u})=\varrho$, where $\varrho$ solves the approximate continuity equation (13) with the Neumann boundary condition. We then claim that the following analogue of Proposition 4.29 from [19], holds true.

Lemma 2.2 Let assumptions of Theorem 2 be satisfied. Then the operator $\mathcal{S}$ is well defined for all $p<\infty$. Moreover, if $\mathcal{S}(\mathbf{u})=\varrho$, then $\varrho \geq 0$ in $\Omega$ and $\int_{\Omega} \varrho \mathrm{d} x=\int_{\Omega} h \mathrm{~d} x$. Additionally, if $\|\mathbf{u}\|_{1, \infty} \leq K, K>0$, then

$$
\|\varrho\|_{2, p} \leq C(\epsilon, p, \Omega)(1+K) h, \quad 1<p<\infty .
$$


Next, the existence of regular solution to the approximate species balance equations is a consequence the following result

Lemma 2.3 Under the hypothesis of previous Lemma there exists a unique solution to (15) $Y_{k} \in W^{2, p}(\Omega)$ for all $1<p<\infty$, such that $Y_{k} \geq 0$ and $\sum_{k \in S} Y_{k}=1$.

Proof. Clearly, in view of regularity of coefficients, the standard theory for elliptic problems yields that $Y_{k} \in W^{2, p}(\Omega)$ for all $1<p<\infty$. Furthermore, the weak maximal principle together with the definition of species production rates $\omega_{k}$ provides suitable bounds from below for each $Y_{k}, k \in S$. Indeed, the operator $\mathcal{L}$ defined through $(15)$ is uniformly elliptic and for $\Omega^{-}=\left\{x \in \Omega: Y_{k}(x)<0\right\}$ we have

$$
\mathcal{L} Y_{k}=0,
$$

thus

$$
\min _{\overline{\Omega^{-}}} Y_{k}=\min _{\partial \Omega^{-}} Y_{k}=0
$$

but this leads to conclusion that $\left|\Omega^{-}\right|=0$ and consequently $Y_{k} \geq 0$ in $\Omega$. SImilarly, one can sum equations (15) together with the conservation of dilutant mass to get that $\sum_{k \in S} Y_{k}=$ const.

Having prepared the necessary information we are ready to handle the last step in the proof of existence of regular solutions. We will use the Leray-Schauder fixed point theorem for the operator

$$
\mathcal{T}: M^{\infty} \rightarrow M^{\infty}
$$

such that $\mathbf{v}=\mathcal{T}(\mathbf{u})$ is a solution of the problem

$$
\begin{gathered}
-\operatorname{div} \mathbb{S}(\mathbf{v})=-\frac{1}{2} \varrho \mathbf{u} \cdot \nabla \mathbf{u}-\frac{1}{2} \operatorname{div}(\varrho \mathbf{u} \otimes \mathbf{u})-\nabla \pi(\varrho, Y)+\varrho \mathbf{f}, \\
\varrho=S(\mathbf{u}), \\
\left.\mathbf{v}\right|_{\partial \Omega}=\mathbf{0} .
\end{gathered}
$$

The existence of unique solution to this system can be shown by the direct application of the Lax-Milgram theorem. Regarding compactness and continuity of operator $\mathcal{T}$, the only difference with respect to the situation studied in [19] is the presence of additional term in the pressure. But, by Lemma 4 one can see that the right hand side is still sufficiently smooth and bounded in $L^{p}(\Omega)$ for $1<p<\infty$ in order to estimate the norm of solution in $W^{2, p}(\Omega)$. The last information we need to verify the hypothesis of the Leray-Schauder fixed point theorem is the boundedness of possible fixed points to

$$
t \mathcal{T}(\mathbf{u})=\mathbf{u}, \quad t \in[0,1]
$$

which will be derived from the first a priori estimate.

Lemma 2.4 Let assumptions of Theorem 2 be satisfied. Let $t \in[0,1], \mathbf{u} \in M^{\infty}$ be a fixed point $\mathbf{u}=t \mathcal{T}(\mathbf{u})$. Then there exists a constant $C>0$ independent of $t \in[0,1]$, such that

$$
\|\mathbf{u}\|_{1,2} \leq C .
$$


Proof. Taking as a test function in (18) the solution $\mathbf{u}$ which satisfies $\mathcal{S}(\mathbf{u})=\varrho$ one gets

$$
\int_{\Omega} \mathbb{S}(\mathbf{u}): \nabla \mathbf{u} \mathrm{d} x=-t \int_{\Omega} \nabla \pi(\varrho, Y) \cdot \mathbf{u} \mathrm{d} x+t \int_{\Omega} \varrho \mathbf{f} \cdot \mathbf{u} \mathrm{d} x .
$$

By definition of $p$ and due to the approximate continuity equation

$$
\begin{array}{r}
\int_{\Omega} \nabla \pi(\varrho, Y) \cdot \mathbf{u} \mathrm{d} x=\gamma \varepsilon \int_{\Omega}|\nabla \varrho|^{2} \varrho^{\gamma-1} \mathrm{~d} x+\frac{\gamma \varepsilon}{\gamma-1} \int_{\Omega} \varrho^{\gamma} \mathrm{d} x-\frac{\gamma h \varepsilon}{\gamma-1} \int_{\Omega} \varrho^{\gamma-1} \mathrm{~d} x \\
-R \int_{\Omega}\left(\sum_{k \in S} \frac{Y_{k}}{m_{k}}\right) \varrho \operatorname{div} \mathbf{u} \mathrm{d} x
\end{array}
$$

hence we may use the Korn inequality to estimate

$$
\begin{aligned}
& (2 \mu+\nu)\|\mathbf{u}\|_{1,2}^{2}+t \gamma \varepsilon \int_{\Omega}|\nabla \varrho|^{2} \varrho^{\gamma-1} \mathrm{~d} x+t \frac{\gamma \varepsilon}{\gamma-1} \int_{\Omega} \varrho^{\gamma} \mathrm{d} x \\
& \leq t \frac{\gamma h \varepsilon}{\gamma-1} \int_{\Omega} \varrho^{\gamma-1} \mathrm{~d} x+t R \int_{\Omega}\left(\sum_{k \in S} \frac{Y_{k}}{m_{k}}\right) \varrho \mathrm{div} \mathbf{u} \mathrm{d} x+t \int_{\Omega} \varrho \mathbf{f} \cdot \mathbf{u} \mathrm{d} x .
\end{aligned}
$$

The first term on the right hand side can be absorbed by the corresponding one on the left hand side. Next, observe that the Hölder and Young inequalities yield

$$
t \int_{\Omega} \frac{Y_{k}}{m_{k}} \varrho \operatorname{div} \mathbf{u} \mathrm{d} x+t \int_{\Omega} \varrho \mathbf{f} \cdot \mathbf{u} \mathrm{d} x \leq \frac{t}{m_{k}}\|\varrho\|_{2}\|\mathbf{u}\|_{1,2}+t\|\mathbf{f}\|_{\infty}\|\varrho\|_{6 / 5}\|\mathbf{u}\|_{1,2}
$$

In order to control the norm of $\varrho$ in $L^{2}(\Omega)$ we test the approximate momentum equation by the function

$$
\Phi=\mathcal{B}\left(\int_{\Omega} \pi^{\beta} \mathrm{d} x-\frac{1}{|\Omega|} \int_{\Omega} \pi^{\beta} \mathrm{d} x\right)
$$

where $\beta \in(0,1]$ and $\mathcal{B}$ is the Bogovskii operator. Recall that (see [1]):

$$
\|\nabla \Phi\|_{p} \leq c(p, \Omega)\left\|\pi^{\beta}\right\|_{p}
$$

and due to the Sobolev imbedding, we have

$$
\begin{aligned}
& \|\Phi\|_{\bar{p}} \leq c(p, \Omega)\left\|\pi^{\beta}\right\|_{p}, \quad 1<p<\infty, \\
& \bar{p}=\left\{\begin{array}{lll}
\frac{3 p}{3-p} & \text { if } & p<3 \\
\in[1, \infty) & \text { if } & p=3 \\
\infty & \text { if } & p>3
\end{array}\right.
\end{aligned}
$$

This testing results in the following identity

$$
\begin{aligned}
\int_{\Omega} \pi^{1+\beta} \mathrm{d} x & =-\frac{1}{2} \int_{\Omega} \varrho(\mathbf{u} \otimes \mathbf{u}): \nabla \Phi \mathrm{d} x+\frac{1}{2} \int_{\Omega} \varrho(\mathbf{u} \cdot \nabla \mathbf{u}) \cdot \Phi \mathrm{d} x \\
& +\int_{\Omega} \mathbb{S}(\mathbf{u}): \nabla \Phi \mathrm{d} x-\int_{\Omega} \varrho \mathbf{f} \cdot \Phi \mathrm{d} x+\frac{1}{|\Omega|} \int_{\Omega} \pi \mathrm{d} x \int_{\Omega} \pi^{\beta} \mathrm{d} x=\sum_{i=1}^{5} I_{i}
\end{aligned}
$$


The "worst" estimate here will be the one connected with the convective term:

$$
I_{1}+I_{2} \leq\|\varrho\|_{(1+\beta) \gamma}\|\mathbf{u}\|_{1,2}^{2}\|\pi\|_{\frac{3 \gamma \beta(1+\beta)}{2 \gamma(1+\beta)-3}}^{\beta} \leq C\|\varrho\|_{(1+\beta) \gamma}^{1+2 a}\|\pi\|_{\beta+1}^{\beta}
$$

where $a$ is the power in the interpolation inequality

$$
\|\varrho\|_{2} \leq\|\varrho\|_{1}^{1-a}\|\varrho\|_{(\beta+1) \gamma}^{a} .
$$

The latter inequality in $(21)$ is true if $\frac{3 \gamma \beta(1+\beta)}{2 \gamma(1+\beta)-3} \leq \beta+1$ meaning that

$$
\beta=\left\{\begin{array}{lll}
\frac{2 \gamma-3}{\gamma} & \text { if } \quad \gamma<3 \\
1 & \text { if } \quad \gamma \geq 3
\end{array}\right.
$$

Hence, from (20) we deduce in particular that independently of $t \in[0,1]$ and $\varepsilon$ we have

$$
\|\varrho\|_{(1+\beta) \gamma} \leq C,
$$

provided $1+2 a<\gamma$. Therefore, by virtue of (22), one can see that the relevant condition on $\gamma$ is $\gamma>\frac{7}{3}$.

Estimate (23), along with (19), leads to the following conclusion

$$
\|\mathbf{u}\|_{1,2}^{2}+t \varepsilon\left(\|\varrho\|_{\gamma}^{\gamma}+\left\|\nabla \varrho^{\frac{\gamma}{2}}\right\|_{2}^{2}\right) \leq C t,
$$

that finishes the proof of Lemma 5.

This information allows us to repeat the procedure described in [19] which together with the Lemmas 3, 4 yield the existence of regular solutions, and hence complete the proof of Theorem 2.

From what has already been computed we get the estimate for the norm of pressure, namely

$$
\|\pi(\varrho, Y)\|_{1+\beta} \leq C
$$

with a constant independent of $\varepsilon$.

Moreover, taking $\varrho$ as a test function in the approximate continuity equation one may get

$$
\sqrt{\varepsilon}\|\nabla \varrho\|_{2} \leq C
$$

Similarly, multiplying the species conservation equations (15) by the $Y_{k}$ and integrating over $\Omega$ we obtain

$$
\int_{\Omega} \operatorname{div}(\varrho \mathbf{u}) \frac{Y_{k}^{2}}{2} \mathrm{~d} x+\int_{\Omega} D_{k}(\varrho)\left|\nabla Y_{k}\right|^{2} \mathrm{~d} x=\int_{\Omega} \varrho \omega_{k} Y_{k} \mathrm{~d} x, \quad k \in\{A, B, C\},
$$

since $0 \leq Y_{k} \leq 1$, and due to (13) we have

$$
\underline{D}\left\|\nabla Y_{k}\right\|_{2}^{2} \leq C\left(\frac{\varepsilon}{2}\|\nabla \varrho\|_{2}\left\|\nabla Y_{k}\right\|_{2}+\varepsilon M+\bar{\omega}\|\varrho\|_{1}\right) .
$$

In view of previous estimates we may thus confirm that $Y_{k}$ is bounded in $W^{1,2}(\Omega)$ for all $k \in S$ independently of $\varepsilon$. 


\section{$3 \quad$ Limit passage to a system with artificial pressure}

The estimates from the previous section can be used to deduce that, at least for a suitable subsequence, we have

$$
\begin{aligned}
\mathbf{u}_{\varepsilon} & \rightarrow \mathbf{u} \text { weakly in } W^{1,2}(\Omega), \\
\varrho_{\varepsilon} & \rightarrow \varrho \text { weakly in } L^{(1+\beta) \gamma}(\Omega), \\
\varepsilon \nabla \varrho_{\varepsilon} & \rightarrow 0 \text { strongly in } L^{p}(\Omega), \quad 1 \leq p \leq 2, \\
\left(Y_{k}\right)_{\varepsilon} & \rightarrow Y_{k} \quad \text { weakly in } W^{1,2}(\Omega), \\
\left(Y_{k}\right)_{\varepsilon} & \rightarrow Y_{k} \text { weakly* in } L^{\infty}(\Omega) .
\end{aligned}
$$

We are hence in a position to conclude that there exists a triple of functions $\left(\varrho, \mathbf{u}, Y_{k}\right)$ that satisfies the integral equalities:

$$
\begin{gathered}
\int_{\Omega} \varrho \mathbf{u} \cdot \nabla \xi \mathrm{d} x=0, \quad \forall \xi \in C^{\infty}(\bar{\Omega}) \\
\int_{\Omega}(-\varrho(\mathbf{u} \otimes \mathbf{u}): \nabla \varphi+\mathbb{S}(\mathbf{u}): \nabla \varphi) \mathrm{d} x-\int_{\Omega} \overline{\pi(\varrho, Y)} \operatorname{div} \varphi \mathrm{d} x=\int_{\Omega} \varrho \mathbf{f} \cdot \varphi \mathrm{d} x \quad \forall \varphi \in C_{0}^{\infty}(\Omega) \\
\int_{\Omega} \varrho \mathbf{u} Y_{k} \nabla \cdot \phi \mathrm{d} x=\int_{\Omega} \overline{D_{k}(\varrho) \nabla Y_{k}} \cdot \nabla \phi \mathrm{d} x-\int_{\Omega} \varrho \omega_{k} \phi \mathrm{d} x, \quad \forall \phi \in C^{\infty}(\bar{\Omega}) .
\end{gathered}
$$

for $k \in\{A, B, C\}$. Here and in the sequel $\overline{g(\varrho, \mathbf{u}, Y)}$ denotes the weak limit of a sequence $g\left(\varrho_{\varepsilon}, \mathbf{u}_{\varepsilon}, Y_{\varepsilon}\right)$.

Accordingly there left two problems that need to be solved, namely, is it true that $\overline{\pi(\varrho, Y)}=\pi(\varrho, Y)$ and is $\overline{D_{k}(\varrho) \nabla Y_{k}}=D_{k}(\varrho) \nabla Y_{k}$ ? As we already have an information about strong convergence of $Y_{k}$ for $k \in S$, the positive answer for the first question is, as will be seen in the sequel, in fact equivalent to the strong convergence of the density. Moreover, having proved this it will be straightforward to see that the second hypothesis holds true as well. It is namely an easy consequence of boundedness of $D_{k}(\varrho)$ in $L^{2}(\Omega)$.

Since $\varrho_{\varepsilon} \mathbf{u}_{\varepsilon}$ and $\nabla \varrho_{\varepsilon}$ possess zero normal traces, it is possible to extend the approximate continuity equation to the whole $\mathbb{R}^{3}$

$$
\varepsilon 1_{\Omega} \varrho_{\varepsilon}+\operatorname{div}\left(1_{\Omega} \varrho_{\varepsilon} \mathbf{u}_{\varepsilon}\right)=\varepsilon \operatorname{div}\left(1_{\Omega} \nabla \varrho_{\varepsilon}\right)+\varepsilon 1_{\Omega} h .
$$

The next step will be based on some properties of the double Riesz transform, defined on the whole $\mathbb{R}^{3}$ in the following way

$$
\mathcal{R}_{i, j}=-\partial_{x_{i}}(-\Delta)^{-1} \partial_{x_{j}},
$$

where the inverse Laplacian is identified through the Fourier transform $\mathcal{F}$ and the inverse Fourier transform $\mathcal{F}^{-1}$ as

$$
(-\Delta)^{-1}(v)=\mathcal{F}^{-1}\left(\frac{1}{|\xi|^{2}} \mathcal{F}(v)\right) .
$$


We will be using general results on such operators as continuity but also some facts concerning the commutators involving Riesz operators, being mostly the consequence of the Coifman-Mayer Lemma [3], [9].

First, we test the approximate momentum equation by the function

$$
\varphi(x)=\zeta(x) \phi, \quad \phi=\left(\nabla \Delta^{-1}\right)\left[1_{\Omega} \varrho_{\varepsilon}\right], \quad \zeta \in C_{0}^{\infty}(\Omega),
$$

observe that this operation "gains" one derivative thus the $L^{(1+\beta) \gamma}(\Omega)$ - integrability of $\varrho_{\varepsilon}$ yields

$$
\left(\nabla \Delta^{-1}\right)\left[1_{\Omega} \varrho_{\varepsilon}\right] \rightarrow\left(\nabla \Delta^{-1}\right)\left[1_{\Omega} \varrho\right] \quad \text { in } C(\bar{\Omega}) .
$$

From this testing we have

$$
\begin{aligned}
& \int_{\Omega} \zeta\left(\pi\left(\varrho_{\varepsilon}, Y_{\varepsilon}\right) \varrho_{\varepsilon}-\mathbb{S}\left(\mathbf{u}_{\varepsilon}\right): \mathcal{R}\left[1_{\Omega} \varrho_{\varepsilon}\right]\right) \mathrm{d} x= \\
& \int_{\Omega} \zeta\left(\varrho_{\varepsilon} \mathbf{u}_{\varepsilon} \cdot \mathcal{R}\left[1_{\Omega} \varrho_{\varepsilon} \mathbf{u}_{\varepsilon}\right]-\varrho_{\varepsilon}\left(\mathbf{u}_{\varepsilon} \otimes \mathbf{u}_{\varepsilon}\right): \mathcal{R}\left[1_{\Omega} \varrho_{\varepsilon}\right]\right) \mathrm{d} x-\int_{\Omega} \zeta \varrho_{\varepsilon} \mathbf{u}_{\varepsilon} \cdot \nabla \Delta^{-1}\left[\operatorname{div} 1_{\Omega} \varrho_{\varepsilon} \mathbf{u}_{\varepsilon}\right] \mathrm{d} x \\
& +\frac{\varepsilon}{2} \int_{\Omega} \zeta\left(\nabla \varrho_{\varepsilon} \cdot \nabla\right) \mathbf{u}_{\varepsilon} \cdot \nabla \Delta^{-1}\left[1_{\Omega} \varrho_{\varepsilon}\right] \mathrm{d} x+\frac{\varepsilon}{2} \int_{\Omega}\left(\nabla \varrho_{\varepsilon} \otimes \mathbf{u}_{\varepsilon}\right): \nabla\left(\zeta \nabla \Delta^{-1}\left[1_{\Omega} \varrho_{\varepsilon}\right]\right) \mathrm{d} x \\
& +\frac{\varepsilon}{2} \int_{\Omega} \zeta\left(\varrho_{\varepsilon}-h\right) \mathbf{u}_{\varepsilon} \cdot \nabla \Delta^{-1}\left[1_{\Omega} \varrho_{\varepsilon}\right] \mathrm{d} x-\int_{\Omega} \varrho_{\varepsilon}\left(\mathbf{u}_{\varepsilon} \otimes \mathbf{u}_{\varepsilon}\right): \nabla \zeta \otimes \Delta^{-1}\left[1_{\Omega} \varrho_{\varepsilon}\right] \mathrm{d} x \\
& \quad+\int_{\Omega} \mathbb{S}\left(\mathbf{u}_{\varepsilon}\right): \nabla \zeta \otimes \nabla \Delta^{-1}\left[1_{\Omega} \varrho_{\varepsilon}\right] \mathrm{d} x-\int_{\Omega} \pi\left(\varrho_{\varepsilon}, Y_{\varepsilon}\right) \nabla \zeta \otimes \nabla \Delta^{-1}\left[1_{\Omega} \varrho_{\varepsilon}\right] \mathrm{d} x \\
& \quad-\int_{\Omega} \mathbf{f} \cdot \varrho_{\varepsilon} \zeta \nabla \Delta^{-1}\left[1_{\Omega} \varrho_{\varepsilon}\right] \mathrm{d} x=\sum_{i=1}^{9} I_{i}
\end{aligned}
$$

where $I_{2}$ may be expressed by means of approximate continuity equation (31) in the following way

$$
I_{2}=-\varepsilon \int_{\Omega} \zeta \varrho_{\varepsilon} \mathbf{u}_{\varepsilon} \cdot \nabla \Delta^{-1}\left[\operatorname{div} 1_{\Omega} \nabla \varrho_{\varepsilon}\right] \mathrm{d} x+\varepsilon \int_{\Omega} \zeta \varrho_{\varepsilon} \mathbf{u}_{\varepsilon} \cdot \nabla \Delta^{-1}\left[1_{\Omega}\left(\varrho_{\varepsilon}-h\right)\right] \mathrm{d} x .
$$

We will compare (33) with a similar expression obtained by testing the limit momentum equation with the function

$$
\varphi(x)=\zeta(x) \phi, \quad \phi=\left(\nabla \Delta^{-1}\right)\left[1_{\Omega} \varrho\right], \zeta \in C_{0}^{\infty}(\Omega),
$$

we have

$$
\begin{gathered}
\int_{\Omega} \zeta\left(\overline{\pi(\varrho, Y)} \varrho-\mathbb{S}(\mathbf{u}): \mathcal{R}\left[1_{\Omega} \varrho\right]\right) \mathrm{d} x=\int_{\Omega} \zeta\left(\varrho \mathbf{u} \cdot \mathcal{R}\left[1_{\Omega} \varrho \mathbf{u}\right]-\varrho(\mathbf{u} \otimes \mathbf{u}): \mathcal{R}\left[1_{\Omega} \varrho\right]\right) \mathrm{d} x \\
-\int_{\Omega} \varrho_{\varepsilon}(\mathbf{u} \otimes \mathbf{u}): \nabla \zeta \otimes \nabla \Delta^{-1}\left[1_{\Omega} \varrho\right] \mathrm{d} x+\int_{\Omega} \mathbb{S}(\mathbf{u}): \nabla \zeta \nabla \Delta^{-1}\left[1_{\Omega} \varrho\right] \mathrm{d} x \\
-\int_{\Omega} \overline{\pi(\varrho, Y)} \nabla \zeta \otimes \nabla \Delta^{-1}\left[1_{\Omega} \varrho\right] \mathrm{d} x-\int_{\Omega} \mathbf{f} \varrho \zeta \nabla \Delta^{-1}\left[1_{\Omega} \varrho\right] \mathrm{d} x=\sum_{i=1}^{5} I_{i} .
\end{gathered}
$$


Now, recalling $(25,26,27,28,29)$ and $(32)$, we get that the $\varepsilon$-dependent integrals on the right hand side of (33) disappear, whence $I_{6}, I_{7}, I_{8}, I_{9}$ converge to their counterparts in (34), hence we are left with

$$
\begin{aligned}
& \lim _{\varepsilon \rightarrow 0} \int_{\Omega} \zeta\left(\pi\left(\varrho_{\varepsilon}, Y_{\varepsilon}\right) \varrho_{\varepsilon}-\mathbb{S}\left(\mathbf{u}_{\varepsilon}\right): \mathcal{R}\left[1_{\Omega} \varrho_{\varepsilon}\right]\right) \mathrm{d} x-\int_{\Omega} \zeta\left(\overline{\pi(\varrho, Y)} \varrho-\mathbb{S}(\mathbf{u}): \mathcal{R}\left[1_{\Omega} \varrho\right]\right) \mathrm{d} x= \\
& \lim _{\varepsilon \rightarrow 0} \int_{\Omega} \zeta\left(\varrho_{\varepsilon} \mathbf{u}_{\varepsilon} \cdot \mathcal{R}\left[1_{\Omega} \varrho_{\varepsilon} \mathbf{u}_{\varepsilon}\right]-\varrho_{\varepsilon}\left(\mathbf{u}_{\varepsilon} \otimes \mathbf{u}_{\varepsilon}\right): \mathcal{R}\left[1_{\Omega} \varrho_{\varepsilon}\right]\right) \mathrm{d} x-\int_{\Omega} \zeta\left(\varrho \mathbf{u} \cdot \mathcal{R}\left[1_{\Omega} \varrho \mathbf{u}\right]-\varrho(\mathbf{u} \otimes \mathbf{u}): \mathcal{R}\left[1_{\Omega} \varrho\right]\right) \mathrm{d} x .
\end{aligned}
$$

Our aim will be to show that the right hand side of the above equivalence disappears. For this purpose we will apply the result being a straightforward consequence of the Div-Curl Lemma

Lemma 3.1 Let

$$
\begin{aligned}
& V_{\varepsilon} \rightarrow V \quad \text { weakly in } L^{p}\left(\mathbb{R}^{3}\right), \\
& r_{\varepsilon} \rightarrow r \quad \text { weakly in } L^{q}\left(\mathbb{R}^{3}\right),
\end{aligned}
$$

where

$$
\frac{1}{p}+\frac{1}{q}=\frac{1}{s}<1
$$

Then

$$
V_{\varepsilon} \mathcal{R}\left(r_{\varepsilon}\right)-r_{\varepsilon} \mathcal{R}\left(V_{\varepsilon}\right) \rightarrow V \mathcal{R}(r)-r \mathcal{R}(V) \text { weakly in } L^{s}\left(\mathbb{R}^{3}\right) .
$$

We take $V_{\varepsilon}=\varrho_{\varepsilon} \mathbf{u}_{\varepsilon}, r_{\varepsilon}=\varrho_{\varepsilon}$ and check that they satisfy the assumptions for $p=\frac{6(1+\beta) \gamma}{(1+\beta) \gamma+6}$, $q=(1+\beta) \gamma$ where by $\varrho_{\varepsilon}, \mathbf{u}_{\varepsilon}, \varrho$, $\mathbf{u}$ we mean the functions extended by 0 outside $\Omega$ to the whole space.

We have enough room to choose $s$ such that $\lim _{\varepsilon \rightarrow 0} \int_{\Omega} \zeta \mathbf{u}_{\varepsilon}\left(V_{\varepsilon} \mathcal{R}\left(r_{\varepsilon}\right)-r_{\varepsilon} \mathcal{R}\left(V_{\varepsilon}\right)\right) \mathrm{d} x$ exists, i.e., we take $\frac{6}{5}<s<\frac{6(1+\beta) \gamma}{(1+\beta) \gamma+12}$. Hence, we finally arrive at

$$
\lim _{\varepsilon \rightarrow 0} \int_{\Omega} \zeta\left(p\left(\varrho_{\varepsilon}, Y_{\varepsilon}\right) \varrho_{\varepsilon}-\mathbb{S}\left(\mathbf{u}_{\varepsilon}\right): \mathcal{R}\left[1_{\Omega} \varrho_{\varepsilon}\right]\right) \mathrm{d} x=\int_{\Omega} \zeta\left(\overline{p(\varrho, Y)} \varrho-\mathbb{S}(\mathbf{u}): \mathcal{R}\left[1_{\Omega} \varrho\right]\right) \mathrm{d} x .
$$

Observe that by the fact that $\zeta \in C_{0}^{\infty}(\bar{\Omega})$ one may integrate by parts the second term on the left hand side and by the continuity of the Riesz operator we get

$$
\int_{\Omega} \zeta(\overline{\pi(\varrho, Y)} \varrho-(2 \mu+\nu) \operatorname{div} \mathbf{u} \varrho) \mathrm{d} x=\int_{\Omega} \zeta(\overline{\pi(\varrho, Y) \varrho}-(2 \mu+\nu) \overline{\operatorname{div} \mathbf{u} \varrho}) \mathrm{d} x
$$

Now, we will exploit this identity by use of the renormalized continuity equation.

Lemma 3.2 Let $\varrho \in L^{p}\left(\mathbb{R}^{3}\right), p \geq 2, \varrho \geq 0$, a. e. in $\Omega, \mathbf{u} \in W_{0}^{1,2}\left(\mathbb{R}^{3}\right)$ satisfy the continuity equation

$$
\operatorname{div}(\varrho \mathbf{u})=0
$$

in the sense of distributions on $\mathbb{R}^{3}$, then the pair $(\varrho, \mathbf{u})$ solves the renormalized continuity equation (12) in the sense of distributions on $\mathbb{R}^{3}$ where $b(\cdot)$ is specified as follows:

$$
\begin{gathered}
b \in C\left([0, \infty) \cap C^{1}((0, \infty)),\right. \\
\lim _{s \rightarrow 0^{+}}\left(s b^{\prime}(s)-b(s)\right) \in \mathbb{R}, \\
\left|b^{\prime}(s)\right| \leq C s^{\lambda}, \quad s \in(1, \infty), \quad \lambda \leq \frac{p}{2}-1 .
\end{gathered}
$$


The proofs of Lemmas 6, 7 can be found e.g. in [9] or [19].

Applying the last Lemma to the limit continuity equation we can verify that the pair of functions $(\varrho, \mathbf{u})$ extended by zero outside of $\Omega$ is a solution to the renormalized continuity equation as specified in Definition 2. Moreover, taking $b(\varrho)=\varrho \ln \varrho$ and $\xi=1$ it can be deduced from (30) that

$$
\int_{\Omega} \varrho \operatorname{div} \mathbf{u} \mathrm{d} x=0 .
$$

To derive similar relation for the approximative system we test (13) with function $\xi=\ln \left(\varrho_{\varepsilon}+\eta\right), \eta>0$. After letting $\eta$ and $\varepsilon$ to 0 respectively, one gets

$$
\int_{\Omega} \overline{\varrho \operatorname{div} \mathbf{u}} \mathrm{d} x \leq 0 \text {. }
$$

Because of this, identity (35) may be transformed into:

$$
\int_{\Omega} \overline{\pi(\varrho, Y)} \varrho \mathrm{d} x \geq \int_{\Omega} \overline{\pi(\varrho, Y) \varrho} \mathrm{d} x
$$

and by definition of $p_{\delta}$ we thus have

$$
\int_{\Omega}\left(\overline{\varrho^{\gamma}} \varrho+\overline{\sum_{k \in S} \frac{Y_{k}}{m_{k}} \varrho}\right) \mathrm{d} x \geq \int_{\Omega}\left(\overline{\varrho^{\gamma} \varrho}+R \overline{R \varrho \sum_{k \in S} \frac{Y_{k}}{m_{k}} \varrho}\right) \mathrm{d} x .
$$

This inequality can be used to show strong convergence of density as soon as one justifies

$$
\overline{\varrho^{\gamma}} \varrho \leq \overline{\varrho^{\gamma} \varrho}, \quad \overline{\varrho \sum_{k \in S} \frac{Y_{k}}{m_{k}}} \varrho \leq \overline{\varrho \sum_{k \in S} \frac{Y_{k}}{m_{k}} \varrho,}
$$

which is true in the first case since $f(\varrho)=\varrho^{p}$ is an increasing function for $p>0$. Regarding the second relation, observe that for $k \in S$ by the strong convergence of $\left(Y_{k}\right)_{\varepsilon}$ in $L^{p}(\Omega)$ for $p<6$ we have

$$
\overline{\varrho Y_{k}} \varrho=Y_{k} \bar{\varrho} \varrho=Y_{k} \varrho^{2}, \quad \text { while } \quad \overline{\varrho Y_{k} \varrho}=Y_{k} \overline{\varrho^{2}} \geq Y_{k} \varrho^{2},
$$

where the latter is a consequence of convexity of function $\varrho^{2}$.

This in turn implies the pointwise convergence for the density as we have weak convergence and a convergence of norm in $L^{\gamma+1}(\Omega)$ and thus, the proof of Theorem 1 is now complete.

Acknowledgements. The author wishes to express her gratitude to Piotr Mucha from University of Warsaw and to Milan Pokorný from Charles University in Prague for suggesting the problem, stimulating conversations and help during preparation of the paper.

The author was supported by the International Ph.D. Projects Programme of Foundation for Polish Science operated within the Innovative Economy Operational Programme 2007-2013 funded by European Regional Development Fund (Ph.D. Programme: Mathematical Methods in Natural Sciences) and partly supported by the MN Grant No N N201 547438. 


\section{References}

[1] M.E. Bogovskii: Solutions of some problems of vector analysis associated with the operators Div and Grad, Trudy Seminara S.L. Soboleva, Akad. Nauk Novossibirsk (1980), 5-40.

[2] G.-Q. Chen, D. Hoff, K. Trivisa: Global solutions to a model for exothermically reacting compressible flows with large discontinuous initial data, Arch. Rational Mech. Anal., 166 (2003), $321-358$.

[3] R. Coifman, Y. Meyer: On commutators of singular integrals and bilinear singular integrals. Trans. Amer. Math. Soc., 212:315-331, 1975.

[4] D. Donatelli, K. Trivisa: On a multidimensional model for the dynamic combustion of compressible reacting flow. Arch. Rational Mech. Anal. 185 (2007) 379-408.

[5] D. Donatelli, K. Trivisa: On the Motion of a Viscous Compressible Radiative-Reacting Gas, Commun. Math. Phys. 265, 463-491 (2006).

[6] B. Ducomet: A model of thermal dissipation for a one-dimensional viscous reactive and radiative gas, Math. Methods and Models in Appl. Sci. 9, 1323-1349 (1999)

[7] E. Feireisl, H. Petzeltová, K. Trivisa: Multicomponent reactive flows: global-in-time existence for large data. Commun. Pure Appl. Anal. 7 (2008), no. 5, 1017-1047.

[8] E. Feireisl: Dynamics of viscous compressible fluids. Oxford Lecture Series in Mathematics and its Applications, 26. Oxford University Press, Oxford, 2004.

[9] E. Feireisl, A. Novotný: Singular limits in thermodynamics of viscous fluids. Advances in Mathematical Fluid Mechanics. Birkhäuser Verlag, Basel, 2009.

[10] V. Giovangigli: Multicomponent Flow Modeling, Birkhäuser, Basel, 1999.

[11] Y.-S. Kwon, K. Trivisa: Stability and large time behaviour for multicomponent reactive flows, Nonlinearity 22 (2009) 2443-2471.

[12] M. Lewicka, P.B. Mucha: On temporal asymptotics for the $p$ th power viscous reactive gas. Nonlinear Anal. 57 (2004), no. 7-8, 951-969.

[13] P.-L. Lions: Mathematical Topics in Fluid Mechanics, Vol 2: Compressible Models. Oxford Lecture Series in Mathematics and its Applications, 10. Oxford Science Publications. The Clarendon Press, Oxford University Press, New York, 1998.

[14] P.B. Mucha, M. Pokorný: On a new approach to the issue of existence and regularity for the steady compressible Navier-Stokes equations. Nonlinearity 19 (2006), no. 8, 1747-1768.

[15] P.B. Mucha, M. Pokorný: On the Steady Compressible Navier-Stokes-Fourier System. Commun. Math. Phys. 288, 349-377 (2009)

[16] P.B. Mucha, M. Pokorný: Weak solutions to equations of steady compressible heat conducting fluids. Mathematical Models and Methods in Applied Sciences Vol. 20, No. 5 (2010) 785-813.

[17] A. Novotný, M.Pokorný: Steady compressible Navier-Stokes-Fourier system for monoatomic gas and its generalizations (2011, accepted to: Journal of Differential Equations).

[18] A. Novotný, M.Pokorný: Weak and variational solutions to steady equations for compressible heat conducting fluids (2011, accepted to: SIAM, Journal of Mathematical Analysis). 
[19] A. Novotný, I. Străskraba: Introduction to the mathematical theory of compressible flow. Oxford Lecture Series in Mathematics and its Applications, 27. Oxford University Press, Oxford, 2004.

[20] M. Pokorný, P. B. Mucha: 3D steady compressible Navier-Stokes equations. Cont. Disc. Dynam. Syst. S 1 (2008) 151-163.

[21] A. Zlotnik: Weak solutions of the equations of motion of a viscous compressible reacting binary mixture: uniqueness and Lipschitz-continuous dependence on data (Russian). Mat. Zametki 75(2), 307-311 (2004). 